\title{
The challenges of HIV self-testing in the Eastern Mediterranean Region
}

\author{
Ismaël Maatouk ${ }^{1,2}$ and Moubadda Assi ${ }^{3}$
}

${ }^{1}$ Department of Dermatology, Clemenceau Medical Center affiliated with Johns Hopkins Beirut, Lebanon ${ }^{2}$ Faculty of Health \& Life Sciences, De Montfort University, Leicester, United Kingdom. 35urveillance Officer, National AIDS Control Program in Lebanon, Beirut, Lebanon. (Correspondence to: I. Maatouk: ismaelmaatouk@gmail.com; ismael.maatouk@cmc.com.lb).

Citation: Maatouk I; Assi M. The challenges of HIV self-testing in the Eastern Mediterranean Region. East Mediterr Health J. 2020;26(8):884-885. https://doi.org/10.26719/emhj.20.088

Received: 20/10/19; accepted: 21/01/20

Copyright (c) World Health Organization (WHO) 2020. Open Access. Some rights reserved. This work is available under the CC BY-NC-SA 3.0 IGO license (https://doi.org/10.26719/emhj.20.088)

\section{Introduction}

There is a global commitment to end AIDS by 2030. In order to achieve this commitment, and in line with the 3rd Sustainable Development Goal, the Joint United Nations Programme on HIV and AIDS (UNAIDS) set 3 measurable targets $^{1}$ to be met by 2020 (1). At the core of these targets resides the importance of HIV testing, which is essential to the ultimate success of HIV response. The World Health Organization (WHO) introduced and adopted HIV self-testing (HIVST) in recent years to scale up testing (2). The objective of this editorial is to discuss potential benefits, limitations and impact of HIVST in the Eastern Mediterranean Region.

\section{Overview of the HIV epidemic in the Eastern Mediterranean Region}

The Eastern Mediterranean Region has recorded the highest regional increase rate in HIV incidence in the past 7 years (3). New HIV infections in the Region reached 36000 in 2017 , marking a $28 \%$ increase on the 29000 infections reported in 2010 (3). Life-saving antiretroviral therapy (ART) is available in the Region but has not been able to prevent AIDS-related mortality. Around 10000 people died of an AIDS-related illness in 2017, marking an $11 \%$ rise since 2010 (3). In the Region, the percentage of HIV infections remained low in the general adult population $(<0.1 \%)$ and high among key populations (4). For instance, 95\% of new HIV infections in the Region are within key populations: people who inject drugs (PWID) and their partners; men who have sex with men (MSM) and their partners; and sex workers (SWs) and their clients (4). Key populations affected by HIV differ across the Region. Higher HIV prevalence rates are found among PWID in Pakistan (21\%) and the Islamic Republic of Iran (9.3\%); among MSM in Lebanon (12.6\%) and Morocco (5.7\%); and SWs in Djibouti (12.9\%) and Somalia (5.2\%). With only $34 \%$ of people living with HIV (PLHIV) aware of their status, there is clearly a gap at the top of the pyramid with regard to access to HIV testing. In addition to key populations, sexual and injecting partners of
PLHIV are at high risk of HIV transmission and should be included in HIV testing to identify PLHIV efficiently. However, a negligible number of partners of PLHIV were tested in 2017 (1).

\section{HIVST recommended by WHO and en- dorsed globally}

HIVST is a process through which a person collects his or her own specimen (oral fluid or blood), performs a test, and interprets its results in a private setting, either alone or with a trusted person (2). This initiative is expected to reach first-time testers and create demand among people that need frequent retesting, such as high-risk populations and serodiscordant couples. Thus, this scale-up in testing should help increase the rate of PLHIV who are aware of their status. Moreover, HIVST represents another step forward in line with efforts to increase patient autonomy and self-care interventions, decentralize services, and create demand for HIV testing among those not reached by existing services (5).

HIVST has 2 major limitations: the inability to track seropositive testers and the confirmatory power of the tool. This method involves a certain level of independence and assumes a basic level of knowledge of HIV to ensure proper interpretation of reactive tests and linkage to further testing and care (6). In fact, interpretation of HIVST results needs to be validated by trained professionals and bold national testing strategies $(2,6)$. To date, 88 countries have implemented HIVST and included it in their national HIV strategy (7).

\section{HIVST implementation in the Eastern Mediterranean Region}

Across the Eastern Mediterranean Region, the usage, efficacy and acceptability of HIVST are under-researched. Two qualitative studies conducted in 2016 interviewed MSM from Tunisia, Lebanon and Morocco, and female SWs and PWID from Jordan, Lebanon, Morocco and Tunisia, and introduced the concept of HIVST $(8,9)$. In both studies, the potential of this approach to contribute to a 
sharp scale-up of testing was acknowledged but concerns were expressed with regards to accuracy of the self-test, fear of self-harm, and absence of trained professionals. Access to HIVST via appropriate and safe delivery channels (internet-based distributors, community centres, non-stigmatizing pharmacies, physician clinics, outreach workers, or peers) were acknowledged to reduce many of these barriers for key populations. The directly assisted approach was preferred over the delivery of HIVST by the majority of PWID and female SWs in Jordan, Lebanon, Morocco and Tunisia, and by some of the MSM participants interviewed from Tunisia, Lebanon and Morocco.

A supportive environment should be ensured before implementing HIVST across the Region. First, regulatory authorities should have data about the targeted key population, mapping, stakeholder mapping and clear community engagement. This will allow programmes to identify which populations will benefit most from selftesting using existing routine indicators for HIV testing and linkage to prevention and treatment. After choosing the HIVST products, national authorities should develop or update their laws, policies and regulations, including the age of consent for HIVST. Additionally, HIVST approaches should be clear to facilitate linkage to further testing, prevention and treatment following self-testing (referral, follow-up, treatment initiation and partner services). Thus, HIV testing guidelines and regulations, standard operating procedures and promotional materials should be developed. Moreover, training on guidelines, policies and regulations should be conducted with relevant communities, regulators, national agencies, implementing partners and healthcare workers. Finally, programmes should update their HIV indicators by adding HIVST-specific monitoring and reporting tools.

In conclusion, HIVST has the potential to play a catalytic role in ending the AIDS epidemic by scaling up testing and maximizing the first of the 3 gos. Its implementation gives an indisputable hope for scaleup of HIV testing, especially in an area like the Eastern Mediterranean Region. Among all WHO Regions, the Eastern Mediterranean might be the most in need of HIV testing scale-up approaches, which will be the occasion to improve HIV monitoring systems. Further research needs to be intensified in the Region to keep up with this ever-dynamic landscape to maximize the potential of HIVST.

\section{References}

1. Hermez J, Khattabi H, Sabry A, Riedner G, Hajjeh R. Achieving the Sustainable Development Goal 3: challenges in HIV testing in the Eastern Mediterranean Region. East Mediterr Health J. 2017 Dec 14;23(10):647-8. http://dx.doi.org/10.26719/2017.23.10.647 PMID:30378672

2. Consolidated guidelines on HIV prevention, diagnosis, treatment and care for key populations - 2016 update. Geneva: World Health Organization; 2016. (https://apps.who.int/iris/bitstream/handle/10665/246200/9789241511124-eng.pdf?sequence=8, accessed 1 July 2020).

3. AIDS and sexually transmitted diseases, World AIDS Day 2018. Cairo: World Health Organization Regional Office for the Eastern Mediterranean; 2018 (http://www.emro.who.int/world-aids-campaigns/wad2018/index.html, accessed 1 July 2020).

4. Maatouk I, Assi M, Hermez J. Partner notification in the Eastern Mediterranean Region: is there a way? East Mediterr Health J. Oct 13;25(9):660-1. http://dx.doi.org/10.26719/emhj.19.076 PMID:31625592

5. WHO consolidated guideline on self-care interventions for health: sexual and reproductive health and rights. Geneva: World Health Organization; 2019 (https://www.who.int/reproductivehealth/publications/self-care-interventions/en/, accessed 27 June 2020).

6. Pai NP, Sharma J, Shivkumar S, Pillay S, Vadnais C, Joseph L, et al. Supervised and unsupervised self-testing for HIV in highand low-risk populations: a systematic review. PLOS Med. 2013;10(4):e1001414. http://dx.doi.org/10.1371/journal.pmed.1001414 PMID:23565066

7. Market and technology landscape: HIV rapid diagnostic tests for self-testing, 4th edition. Geneva: Unitaid; 2018 (https://unitaid. org/assets/HIV-Rapid-Diagnostic-Tests-for-Self-Testing_Landscape-Report_4th-edition_July-2018.pdf, accessed 27 June 2020).

8. Guidelines on HIV self-testing and partner notification: supplement to consolidated guidelines on HIV testing services. Supplement to consolidated guidelines on HIV testing services. Annex 30. Report on the values and preferences on HIV self-testing and partner notification in Jordan, Lebanon, Morocco and Tunisia. Geneva: World Health Organization; 2016 (https://www.who.int/ hiv/pub/vct/Annex30.pdf?ua=1, accessed 27 June 2020).

9. Guidelines on HIV self-testing and partner notification: supplement to consolidated guidelines on HIV testing services. Annex 31. Community values and preferences regarding HIV self-testing and partner notification in Tunisia, Lebanon and Morocco: men who have sex with men, transgender people and people living with HIV. Geneva: World Health Organization; 2016 (https:// www.who.int/hiv/pub/vct/Annex31.pdf?ua=1, accessed 27 June 2020). 